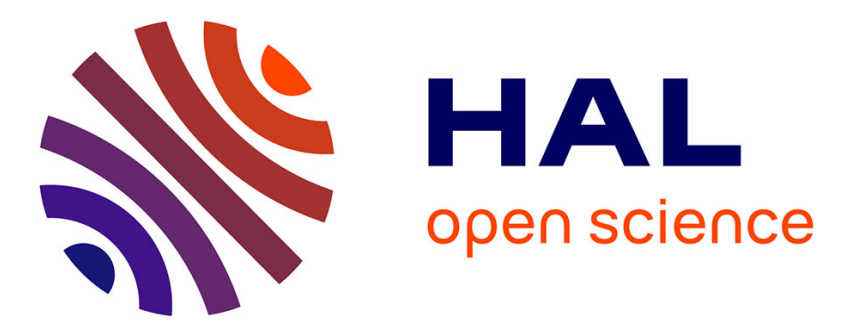

\title{
Analyse radiocristallographique de la distorsion magnétostrictive dans les antiferromagnétiques KCof3, RbCof3 et TlCof3
}

\author{
J. Julliard, J. Nouet
}

\section{- To cite this version:}

J. Julliard, J. Nouet. Analyse radiocristallographique de la distorsion magnétostrictive dans les antiferromagnétiques KCof3, RbCof3 et TlCof3. Revue de Physique Appliquée, 1975, 10 (5), pp.325-329. 10.1051/rphysap:01975001005032500 . jpa-00243920

\section{HAL Id: jpa-00243920 https://hal.science/jpa-00243920}

Submitted on 1 Jan 1975

HAL is a multi-disciplinary open access archive for the deposit and dissemination of scientific research documents, whether they are published or not. The documents may come from teaching and research institutions in France or abroad, or from public or private research centers.
L'archive ouverte pluridisciplinaire HAL, est destinée au dépôt et à la diffusion de documents scientifiques de niveau recherche, publiés ou non, émanant des établissements d'enseignement et de recherche français ou étrangers, des laboratoires publics ou privés. 


\title{
ANALYSE RADIOCRISTALLOGRAPHIQUE DE LA DISTORSION MAGNÉTOSTRICTIVE DANS LES ANTIFERROMAGNÉTIQUES $\mathrm{KCOF}_{3}, \mathrm{RbCoF}_{3}$ ET $\mathrm{TICoF}_{3}$
}

\section{J. JULLIARD et J. NOUET}

Laboratoire de Physique de l'Etat Condensé, Faculté des Sciences Route de Laval, 72000 Le Mans Cedex 72017, France

(Reşu le 4 février 1975, révisé le 10 avril 1975, accepté le 14 avril 1975)

\begin{abstract}
Résumé. - Un cryostat à hélium liquide a été adapté sur un goniomètre à rayons $\mathrm{X}$ afin de permettre la mesure continue des paramètres cristallins de $4 \mathrm{~K}$ à $300 \mathrm{~K}$. Une distorsion quadratique $(c / a<1)$ est mise en évidence dans plusieurs pérovskites fluorées du cobalt ; elle apparaît à $117 \mathrm{~K}$ dans $\mathrm{KCoF}_{3}, 101 \mathrm{~K}$ dans $\mathrm{RbCoF}_{3}$ et $94 \mathrm{~K}_{\text {dans }} \mathrm{TlCoF}_{3}$. La température de transition coïncide avec la température de Néel déterminée par diffraction de neutrons. Le caractère magnétostrictif de la transition est confirmé par l'étude comparée, en fonction de la température, du carré de l'aimantation et de l'amplitude de la distorsion.
\end{abstract}

\begin{abstract}
A liquid helium cryostat has been adapted for an X-ray goniometer in order to allow measurements of lattice constants from 4 to 300 K.A tetragonal distortion $(c / a<1)$ is observed in three cobalt fluoperovskites compounds : below $117 \mathrm{~K}$ in $\mathrm{KCoF}_{3}, 101 \mathrm{~K}$ in $\mathrm{RbCoF}_{3}$ and $94 \mathrm{~K}$ in $\mathrm{TlCoF}_{3}$. The phase transition temperature coincides with the Néel temperature obtained from neutron diffraction. The temperature dependence of $\frac{c-a}{a}$ compared with the temperature dependence of $\mathbf{M}_{z}^{2}$ allows us to confirm the magnetostrictive character of this distortion.
\end{abstract}

1. Introduction. - L'étude de la structure des matériaux à basse température nécessite une analyse radiocristallographique afin de déterminer les paramètres cristallins et de rechercher l'existence d'éventuelles distorsions sur une large gamme de températures. Plus précisément, dans le cas de changements de phase liés à un ordre magnétique, il est intéressant de suivre l'évolution progressive de la distorsion afin de relier celle-ci au paramètre d'ordre obtenu sur le même échantillon par une autre technique (diffraction de neutrons par exemple).

Dans ce but, nous avons adapté sur un goniomètre à rayons $\mathrm{X}$ un cryostat prototype à température variable mis au point par la société M. E. R. I. C. Nous étudions ici la distorsion quadratique qui apparaît en même temps que l'ordre antiferromagnétique dans les matériaux à structure pérovskite $\mathrm{KCoF}_{3}, \mathrm{RbCoF}_{3}$ et $\mathrm{TlCoF}_{3}$.

2. Description et étalonnage du dispositif. 2.1 PRINCIPE. - L'échantillon est refroidi par convection dans un courant d'hélium prélevé en phase liquide ou gazeuse dans la réserve du cryostat. Comme l'indique le schéma synoptique de la figure 1, la circulation du fluide réfrigérant est établie par pompage sur le canal central où est placé l'échantillon en poudre

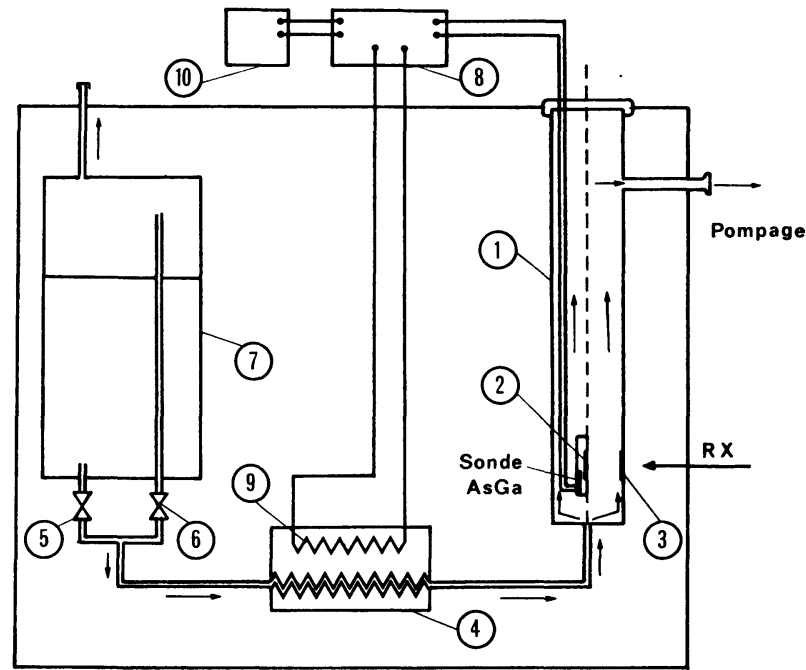
(1) Canal central
(6) Vanne gaz
(2) Echantillont
(7) Réserve hélium liquide
(3) Fenêtre de béryllium
(8) Régulateur
(4) Echangeur
(5) Vanne liquide
(9) Résistance chauffante
(10) Voltmètre

FIG. 1. - Schéma synoptique du cryostat à hélium liquide pour diffractométrie $\mathrm{X}$ à basse température. 
à étudier. Un échangeur, dont la température peut être régulée par chauffage d'un thermocoax incorporé, permet de fixer la température du courant d'hélium. Pour des températures supérieures à $70 \mathrm{~K}$, l'azote liquide plus économique peut utilement remplacer l'hélium liquide.

2.2 Description. Le CRyostat est CONSTitué (Fig. 2). - D'une enveloppe extérieure dont la partie inférieure est démontable et comporte une fenêtre en béryllium d'épaisseur $0,2 \mathrm{~mm}$ pour le passage des rayons $X$. La partie inférieure sert de support au cryostat et présente une table micrométrique permettant la mise en coïncidence du plan de l'échantillon avec l'axe du goniomètre.

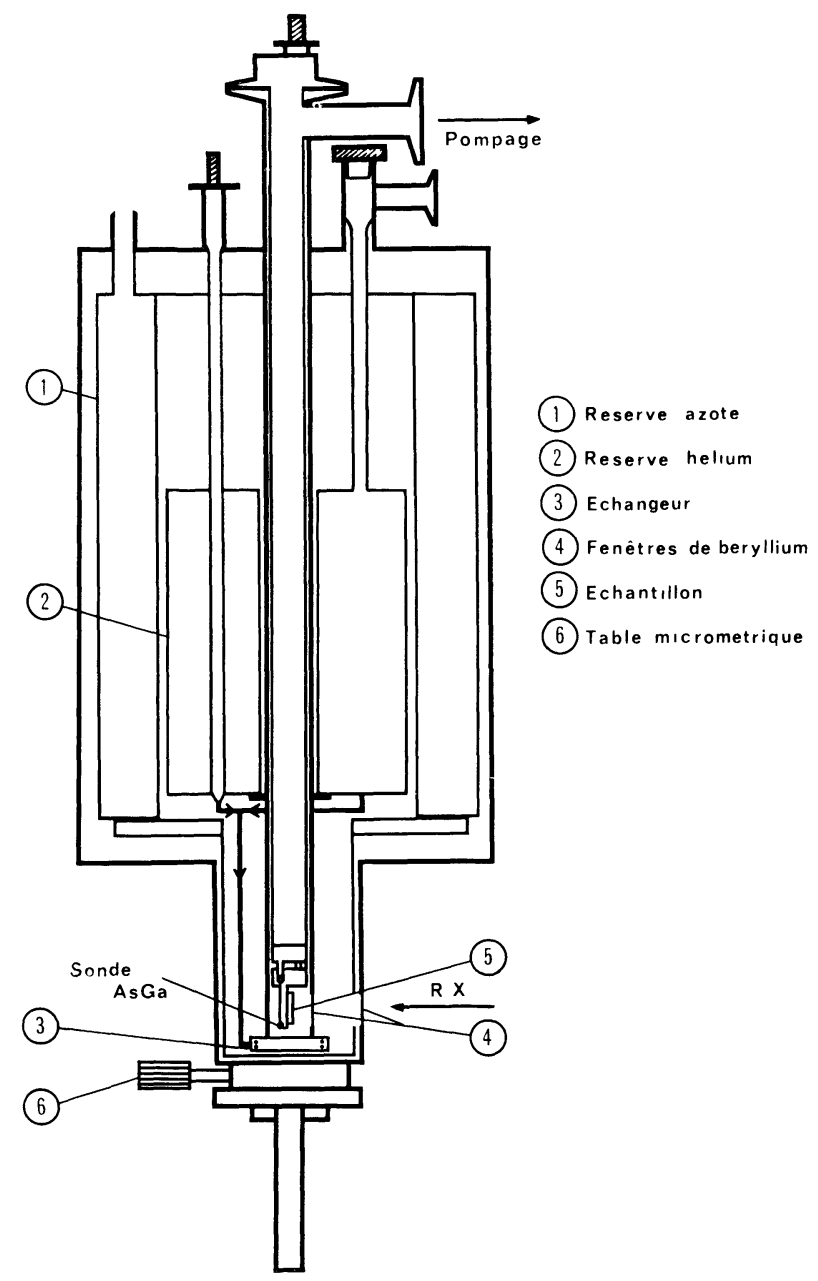

FIG. 2. - Schéma du cryostat M. E. R. I. C. vu en coupe.

- D'une réserve d'hélium liquide avec deux tiges de commande permettant de régler le débit du fluide en phase liquide ou gazeuse. Elle est entourée d'une garde d'azote liquide afin d'assurer l'isolement thermique.

- D'un canal central avec une fenêtre de béryllium et à sa base l'échangeur de grande inertie thermique.

- D'un dispositif de fixation et de commande de la plaquette porte-échantillon adaptable au canal central. Il permet de régler le parallélisme du plan de l'échantillon avec l'axe du goniomètre.
Un régulateur M. V. 2000 (licence ANVAR) de type P. I. D. alimente la résistance de chauffage incorporée dans l'échangeur. Il est commandé par une sonde AsGa fixée sur la plaque support de l'échantillon.

2.3 Etalonnage. - La sonde thermométrique à l'arséniure de gallium est étalonnée en plusieurs points de la température ambiante jusqu'à la température de l'hélium liquide. En cours de régulation, les variations de température sont toujours inférieures à $0,1 \mathrm{~K}$ pour toute température comprise entre 4 et $300 \mathrm{~K}$. La sonde est en contact direct avec le porte-échantillon, il en résulte ainsi un très faible écart entre la température lue et celle de l'échantillon en poudre.

Nous avons vérifié cet étalonnage en déterminant la température de la première transition de type antiferrodistorsif dans $\mathrm{KMnF}_{3}$. Cette transition a déjà donné lieu à de nombreuses études, en particulier par O. Beckman et K. Knox [1], A. Okazaki et Y. Suemune [2], ou V. J. Minkiewicz et coll. [3]. Notre étude effectuée sur anticathode de cuivre permet de situer la température de la transition cubique-quadratique $(c / a>1)$ à $184 \mathrm{~K}$ conformément aux résultats déjà publiés.

A chaque température, la mesure des paramètres cristallins est effectuée par référence à un composé étalon connu (silicium ou diamant) après correction de divergence et de constante de temps. Les variations observées du paramètre cristallin de $\mathrm{KMnF}_{3}$ sont en accord avec les précédents résultats.

3. Etude de la distorsion magnétostrictive dans les fluopérovskites du cobalt : $\mathrm{KCoF}_{3}, \mathrm{RbCoF}_{3}$ et $\mathrm{TICoF}_{3}$. 3.1 Etude RADIOCRISTALlographiQue. - A température ambiante, les composés fluorés $\mathrm{KCoF}_{3}$, $\mathrm{RbCoF}_{3}$ et $\mathrm{TlCoF}_{3}$ sont cubiques du type pérovskite [4] (groupe d'espace $\mathrm{Pm} 3 \mathrm{~m}, \mathrm{O}_{\mathrm{h}}^{1}$ ). Ils ont été préparés par voie sèche à partir du fluorure de cobalt $\mathrm{CoF}_{2}$ et des fluorures alcalins correspondants $\mathrm{KF}, \mathrm{RbF}$ ou TlF. La synthèse par réaction en phase solide dans des conditions de totale déshydratation nous assure d'une bonne stœchiométrie. Ainsi les valeurs des paramètres cristallins sont confirmées par les mesures que nous avons également effectuées sur monocristaux finement broyés [5]. Nous obtenons à $20^{\circ} \mathrm{C}$ :

$$
\begin{array}{lll}
a_{c}=4,0690 \pm 0,0005 \AA & \text { pour } & \mathrm{KCoF}_{3}, \\
a_{c}=4,1270 \pm 0,0005 \AA & \text { pour } & \mathrm{RbCoF}_{3}, \\
a_{c}=4,1440 \pm 0,0005 \AA & \text { pour } & \mathrm{TlCoF}_{3} .
\end{array}
$$

Les écarts observés par rapport aux résultats antérieurs [4] peuvent vraisemblablement être attribués à des défauts de stœechiométrie que l'on peut attribuer à l'utilisation de l'hydrogénofluorure (AF, HF) au cours de la réaction en phase solide.

Le cryostat précédemment décrit nous a permis de compléter sur $\mathrm{KCoF}_{3}$ et $\mathrm{TlCoF}_{3}$ l'étude de $\mathrm{RbCoF}_{3}$ effectuée par J. Nouet, R. Kleinberger et R. de Kouchkovsky [6] à l'aide d'un cryostat M. R. C. de conception différente (Laboratoire de Physique du Solide, C. E. N. Saclay). Pour $\mathrm{KCoF}_{3}$ la distorsion a déjà été 
mise en évidence sur monocristal par A. Okazaki et Y. Suemune [2]. L'étude radiocristallographique sur anticathode de cobalt révèle l'existence à basse température d'une distorsion quadratique caractérisée par $c / a<1$. En effet, les raies de type hhh ne sont pas affectées alors que les raies hhl sont dédoublées, la raie de petit angle ayant une intensité double de l'autre. La détermination du rapport $c / a$ a été effectuée à différentes températures en étudiant la raie 400 située (à $295 \mathrm{~K}$ ) à $122,96^{\circ}(2 \theta)$ pour $\mathrm{KCoF}_{3}$ et à $119,36^{\circ}$ (2 $\theta)$ pour $\mathrm{TlCoF}_{3}$. Une telle raie donne naissance dans la phase quadratique à deux raies affines dont l'écart angulaire atteint $0,76^{\circ}(2 \theta)$ pour $\mathrm{KCoF}_{3}$ et $0,72^{\circ}(2 \theta)$ pour $\mathrm{TlCoF}_{3}$ à la température de l'hélium liquide.

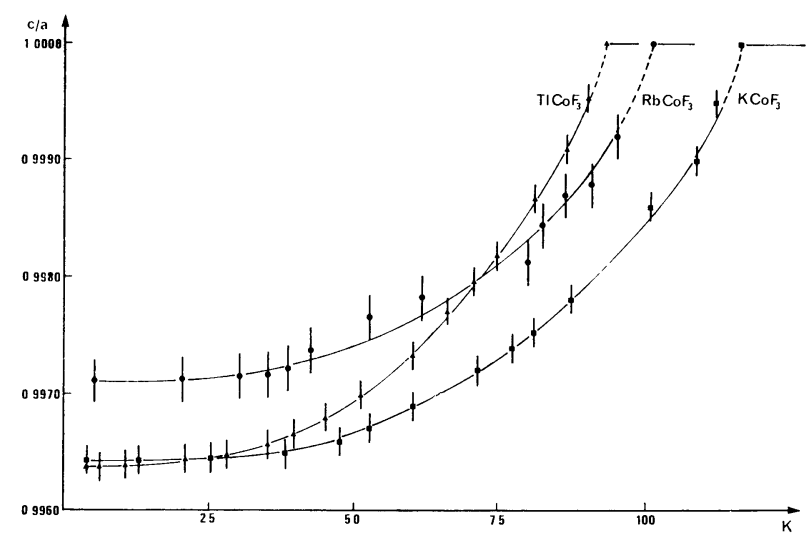

FIG. 3. - Variations de $c / a$ avec la température pour $\mathrm{KCoF}_{3}$, $\mathrm{RbCoF}_{3}$ et $\mathrm{TlCoF}_{3}$.

Sur la figure 3 sont représentées les variations de $c / a$ en fonction de la température. On peut remarquer une évolution analogue pour $\mathrm{RbCoF}_{3}$ et $\mathrm{KCoF}_{3}$ tandis que pour $\mathrm{TlCoF}_{3}$ la variation de $c / a$ est beaucoup plus rapide au voisinage de la transition. Nous pouvons attribuer ce comportement à la présence de l'ion thallium qui est plus facilement déformable que les ions alcalins.

Les mesures ont été effectuées en faisant croître et décroître la température au voisinage de la transition : nous n'avons pas mis en évidence de retard à la transformation. L'étude de la largeur à mi-hauteur de la raie 420 située (à $293 \mathrm{~K}$ ), pour $\mathrm{KCoF}_{3}$, à $158,90^{\circ}(2 \theta)$, pour $\mathrm{TlCoF}_{3}$ à $143,62^{\circ}(2 \theta)$, nous a permis de déterminer avec précision les températures de transition (Fig. 5). Nous n'avons pas observé de discontinuité de volume à la transition (Fig. $4 a$ et $4 b$ ). A la précision des mesures effectuées, nous pouvons considérer que la transformation cristallographique est du second ordre.

Les résultats obtenus relatifs aux trois pérovskites fluorées du cobalt sont regroupés sur la figure 5. Nous observons que la température de transition est d'autant plus élevée que le paramètre cristallin à température ambiante est plus petit. Une telle propriété peut être reliée à l'apparition de l'ordre antiferromagnétique. Aussi, des études magnétiques ont-elles été effectuées en collaboration avec l'équipe du Laboratoire de Physique du Solide du C. E. N., Saclay [8, 9].
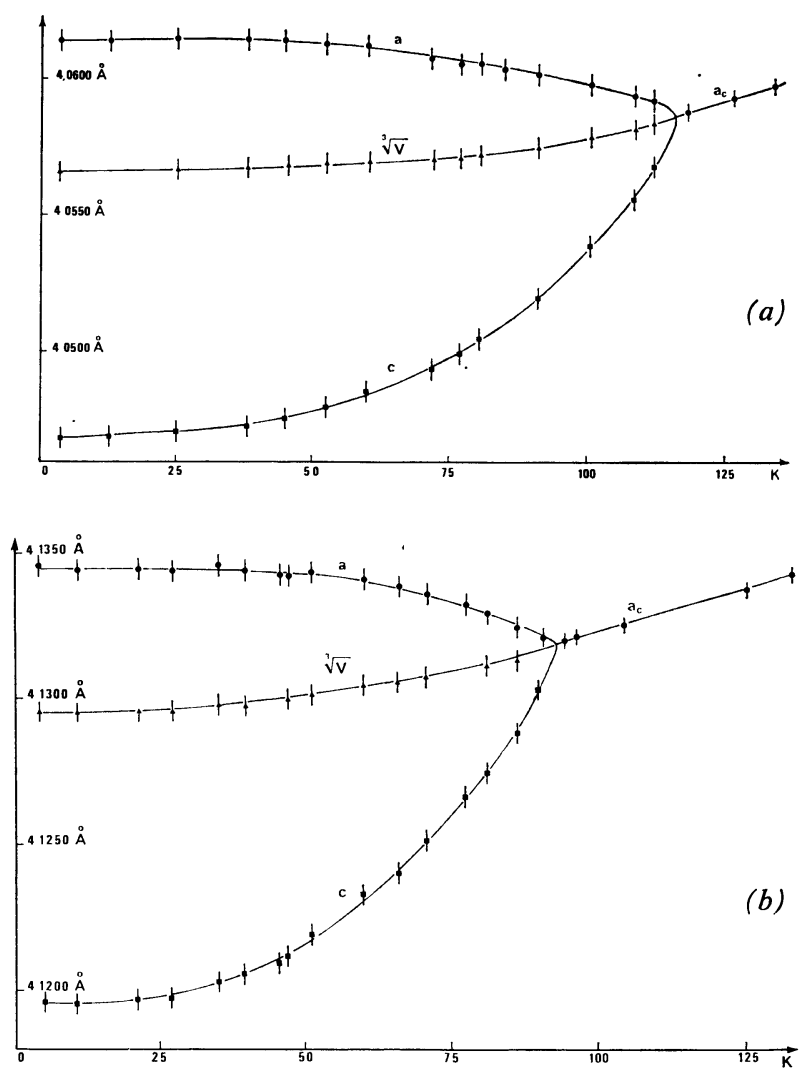

Fig. 4. - Variation, en fonction de la température, des paramètres quadratiques $a$ et $c$, du paramètre moyen quadratique $\sqrt[3]{V}\left(V\right.$, volume de la maille) et du paramètre cubique $a_{\mathrm{c}}:(a)$ pour $\mathrm{KCoF}_{3}$, (b) pour $\mathrm{TlCoF}_{3}$.

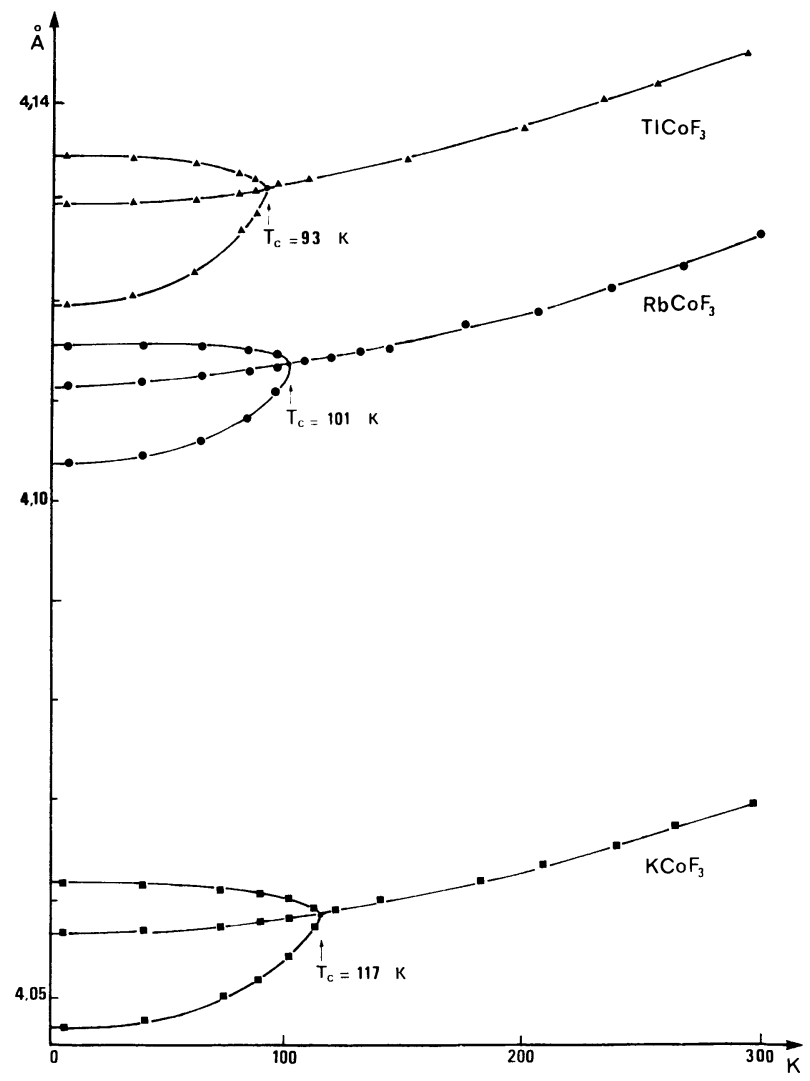

FIG. 5. - Variation des paramètres cristallins avec la température dans $\mathrm{KCoF}_{3}, \mathrm{RbCoF}_{3}$ et $\mathrm{TlCoF}_{3}$. 
3.2 DisTORSION ET ORDRE MAGNÉTIQUE. - Le spectre de diffraction de neutrons montre qu'à $4,2 \mathrm{~K}$ le réseau des atomes de cobalt s'ordonne antiferromagnétiquement suivant une configuration de type $G[7,8,9]$. A partir d'une étude en fonction de la température de la raie magnétique $111_{\mathrm{M}}$, les températures de Néel sont repérées après extrapolation à $T_{\mathrm{N}}=98 \pm 5 \mathrm{~K}$ pour $\mathrm{RbCoF}_{3}$ [8] et $T_{\mathrm{N}}=96 \pm 3 \mathrm{~K}_{\text {pour }} \mathrm{TlCoF}_{3}$ [9]. En raison de l'existence d'un seul pic $\lambda$, les mesures de chaleur spécifique ont permis de conclure à la superposition de la distorsion quadratique et de l'ordre antiferromagnétique à $T_{\mathrm{N}}=101 \pm 1 \mathrm{~K}_{\text {pour }} \mathrm{RbCoF}_{3}$ [8] et $T_{\mathrm{N}}=94 \pm 1 \mathrm{~K}$ pour $\mathrm{TlCoF}_{3}$ [9].

Dans l'hypothèse d'une déformation purement magnétostrictive, les composantes de la déformation peuvent s'exprimer en fonction des constantes $B_{1}$ et $B_{2}$ du couplage magnétoélastique, des cosinus directeurs $\alpha_{i}$ de l'aimantation et des constantes élastiques. Afin d'annuler l'énergie magnétoélastique de dilatation dans la phase non ordonnée magnétiquement où $\alpha_{i}^{2}=\frac{1}{3}$, nous prenons une expression de l'énergie magnétoélastique de la forme :

$$
\begin{aligned}
E_{\mathrm{ME}}=B_{1}\left[e_{11}\left(\alpha_{1}^{2}-\frac{1}{3}\right)+e_{22}\left(\alpha_{2}^{2}-\frac{1}{3}\right)+e_{33}\left(\alpha_{3}^{2}-\frac{1}{3}\right)\right]+ & \\
& +B_{2}\left[e_{12} \alpha_{1} \alpha_{2}+e_{23} \alpha_{2} \alpha_{3}+e_{31} \alpha_{3} \alpha_{1}\right] .
\end{aligned}
$$

En imposant que la somme de cette énergie et de l'énergie élastique soit minimale, nous obtenons les composantes de la déformation :

$$
\begin{aligned}
& e_{i i}=B_{1} \frac{\left(\frac{1}{3}-\alpha_{i}^{2}\right)}{C_{11}-C_{12}} \\
& e_{i j}=-B_{2} \frac{\alpha_{i} \alpha_{j}}{C_{44}} .
\end{aligned}
$$

Les coefficients $B_{1}$ et $B_{2}$ ont été calculés en tenant compte des paramètres du réseau cristallin et des caractéristiques propres à l'ion cobalt dans un champ cristallin cubique. Nous avons pour cela utilisé la méthode de Kanamori déjà appliquée au cas de l'oxyde de cobalt $\mathrm{CoO}$ où l'on observe le même phénomène de distorsion magnétostrictive [10].

L'étude sur monocristal du dichroïsme linéaire sous contrainte [11] a permis de montrer qu'à basse température les spins sont orientés suivant les directions $<001>$. Ainsi, au zéro absolu, la déformation atteint son maximum d'amplitude :

$$
e_{z z}=\left(\frac{c-a_{\mathrm{m}}}{a_{\mathrm{m}}}\right)_{\max }=-\frac{2 B_{1}}{3\left(C_{11}-C_{12}\right)}
$$

avec

$$
a_{\mathrm{m}}=\sqrt[3]{a^{2} c} .
$$

A la température de l'hélium liquide la valeur $\frac{c-a_{\mathrm{m}}}{a_{\mathrm{m}}}$ observée pour $\mathrm{KCoF}_{3}\left(-3 \times 10^{-3}\right)$ est $\mathrm{du}$ même ordre de grandeur mais sensiblement plus faible que la valeur calculée $\left(-5 \times 10^{-3}\right)$. D'autre part, dans l'hypothèse de faibles variations avec la température de $C_{11}-C_{12}$ dans la phase antiferromagnétique, l'amplitude de la distorsion $\frac{c-a}{a}$ et le carré de l'aimantation $M^{2}(T)$ sont liés par une loi linéaire :

$$
\frac{c-a}{a}=\frac{3 B_{1}}{2} \frac{\frac{1}{3}-\alpha_{z}^{2}}{C_{11}-C_{12}}
$$

avec

$$
\alpha_{z}^{2}=\frac{\left\langle M_{z}^{2}>\right.}{M_{0}^{2}}=\frac{M^{2}(T)}{M_{\max }^{2}(0)} .
$$

Cette relation s'écrit après normalisation :

$$
\frac{\frac{c-a}{a}(T)}{\left(\frac{c-a}{a}\right)_{\mathrm{m}}}=\frac{3}{2}\left[\frac{M^{2}(T)}{M_{\max }^{2}}-\frac{1}{3}\right]
$$

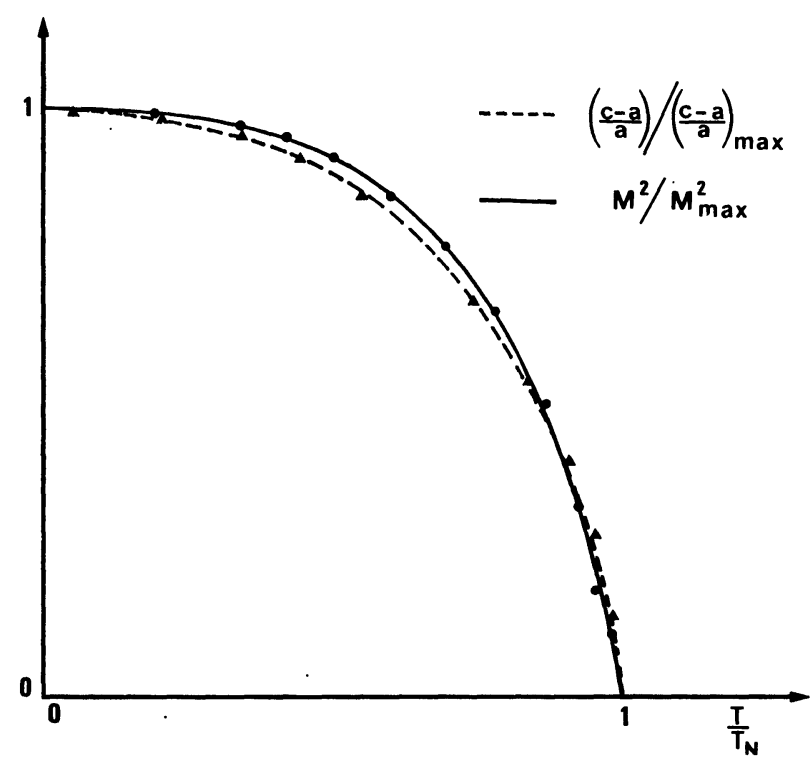

Fig. 6. - Variations avec la température de la distorsion et du carré de l'aimantation après normalisation dans $\mathrm{RbCoF}_{3}$.

elle est vérifiée expérimentalement comme le montre, pour $\mathrm{RbCoF}_{3}$, la figure 6 qui représente les variations normalisées de la distorsion et du carré de l'aimantation en fonction de la température. La courbe $M^{2}(T) / M_{\max }^{2}$ est déduite de l'étude de l'intensité de la raie magnétique $111_{\mathrm{M}}$ du spectre de diffraction de neutrons [8]. On peut donc dire que la déformation quadratique est de type magnétostrictif, elle est étroitement liée à la direction d'aimantation et à l'état d'ordre de chaque sous-réseau. La distorsion de l'ensemble du cristal relève d'un mécanisme coopératif qui ne se manifeste que lorsque l'agitation thermique et les distances entre ions magnétiques deviennent suffisamment faibles pour que le couplage antiferromagnétique se produise entre ions voisins. 
4. Conclusion. - L'appareillage utilisé permet de déterminer avec une bonne précision les coefficients de dilatation d'un matériau polycristallin ou, dans le cas d'un changement de phase, d'étudier la loi de variation de l'amplitude de déformation sur une large gamme de températures. Pour les antiferromagnétiques étudiés, nous avons montré que la distorsion observée est de type essentiellement magnétostrictif. Le fort couplage spin-réseau pouvant être attribué à la nature de l'ion cobalt dont le moment cinétique orbital n'est pas bloqué par le champ cristallin.

Remerciements. - Nous remercions M. R. Kleinberger du Laboratoire de Physique du Solide du C. E. N. Saclay pour ses précieux conseils dans le domaine de la radiocristallographie sur compteur à basse température.

\section{Bibliographie}

[1] Beckman, O., Knox, K., Phys. Rev. 121 (1961) 376.

[2] Okazaki, A. Suemune, Y., J. Phys. Soc. Japan 16 (1961) 671.

[3] Minkiewicz, V. J., FujiI, Y., Yamada Y., J. Phys. Soc. Japan 28 (1970) 443.

[4] Rudorff, W., Lincke, G., Babel, D., Z. Anorg. Allag. Chem. 320 (1963) 150

[5] Nouet, J., Jacoboni, C., Ferey, G., Gerard, J. Y., DE RAPE, R., J. Cryst. Growth 8 (1971) 94.

[6] Nouet, J., Kleinberger, R., DE Kouchkovsky, R., C. R. Hebd. Séan. Acad. Sci. B 269 (1969) 986.
[7] Scatturin, V., Corliss, L., Elliot, N., Hastings, J., Acta Cryst. 14 (1961) 19.

[8] Allain, Y., Denis, J., Herpin, A., Lecomte, J., Meriel, P., Nouet, J., Plicque, F., Zarembowitch, A., J. Physique Colloq. 32 (1971) C 1-611.

[9] Lecomte, M., Nouet, J., Portes, M., C. R. Hebd. Séan. Acad. Sci. B 276 (1973) 579.

[10] Kanamori, J., Prog. Theor. Phys. 17 (1957) 197.

[11] Ferre, J., Briat, B., Pisarev, R. V., Nouet, J., Proceed. Int. Conf. Magn., Moscou, 1973. 